\title{
N-Fold Darboux Transformation for the Nonlocal Nonlinear Schrödinger (NNLS) Equation with the Self-Induced PT-Symmetric Potential
}

\author{
Chaonan Duan, Fajun Yu* \\ School of Mathematics and Systematic Sciences, Shenyang Normal University, Shenyang, China \\ Email: *yfajun@163.com
}

How to cite this paper: Duan, C.N. and Yu, F.J. (2018) N-Fold Darboux Transformation for the Nonlocal Nonlinear Schrödinger (NNLS) Equation with the Self-Induced PT-Symmetric Potential. Journal of Applied Mathematics and Physics, 6, 888-900. https://doi.org/10.4236/jamp.2018.64076

Received: March 22, 2018

Accepted: April 24, 2018

Published: April 27, 2018

Copyright ( 92018 by authors and Scientific Research Publishing Inc. This work is licensed under the Creative Commons Attribution International License (CC BY 4.0).

http://creativecommons.org/licenses/by/4.0/

\begin{abstract}
The Darboux transformation (DT) method is studied in a lot of local equations, but there are few of work to solve nonlocal equations by DT. In this letter, we solve the nonlocal nonlinear Schrödinger equation (NNLSE) with the self-induced PT-symmetric potential by DT. Then the N-fold DT of NNLSE is derived with the help of the gauge transformation between the Lax pairs. Then we derive some novel exact solutions including the bright soliton, breather wave soliton. In particularly, the dynamic features of one-soliton, two-soliton, three-soliton solutions and the elastic interactions between the two solitons are displayed.
\end{abstract}

\section{Keywords}

Nonlocal Nonlinear Schrödinger Equation, N-Fold Darboux Transformation, Lax Pairs, Exact Solution

\section{Introduction}

The Schrödinger (NLS) equation is second-order differential equation of combining material waves and waves, which can be used to describe the optical pulse in the dispersion. The nonlinear Schrödinger equation can discuss many problems, such as one-dimensional self-modulation of monochromatic, self trapping phenomena in nonlinear optics, thermal pulse propagation in solids, the laser beam in atomic Bose-Einstein condensation effect, etc. In a local nonlinear medium, the intensity of light at a certain point will only change the refractive index of this point, but the refractive index of a point in the nonlocal medium will 
not only affects the refractive index of the point, but also affects the refractive index of the medium around a certain range. In many natural phenomena, nonlocality is a universal phenomenon. Some theoretical analysis and numerical simulation show that the nonlocality can eliminate the collapse of the wave and greatly improve the interaction between the dark solitons. Under a single response limit, the interaction between waves and media is often described by nonlocal nonlinear Schrödinger equation. This equation has been widely used in various fields of nonlinear physics. Spatial soliton in nonlocal nonlinear media has been paid attention to because of its rich characteristics and important potential application value in nonlocal media. In 2013, Ablowitz and Musslimani proposed PT-symmetric nonlocal nonlinear Schrödinger (NNLS) equation [1] [2] [3], and gave the dark and anti-dark soliton interaction by using the Darboux transformation (DT) method [4] [5] [6].

Soliton theory becomes one of the main theories for studying nonlinear equation, and it also produces various methods to solve soliton equations, such as the inverse scattering method [7] [8] [9], the nonlinear method [10] [11], Hirota bilinear method [12] [13], the homogeneous balance method [14] [15], Bäcklund transformation method [16] [17], DT method [18] [19], etc. For example, the relevant problems of Sine-Gordon equation and pseudo spherical DT have already appeared in [20], it obtains the exact solution of soliton equation from the trivial solution, and it is a very effective method. Gu Chaohao proposed the reversibility of the DT [21]. Fan Engui obtained exact solutions of G-I equation by DT [22]. The DT method analytic representation of the K-E equation is constructed by Qiu Deqin. Some discrete rogue-wave solutions with dispersion in parity-time symmetric potential of Ablowitz-Musslimani equation are derived in [23]. Some bright, dark and breather wave soliton solutions of the super-integrable hierarchy are derived using DT [24]. The non-autonomous multi-rogue wave solutions in a spin-1 coupled nonlinear Gross-Pitaevskii equation with varying dispersions, higher nonlinearities, gain/loss and external potentials are investigated in [25]. Several non-autonomous matter-wave solitons of the generalized three-coupled Gross-Pitaevskii equations are obtained in [26]. The non-autonomous discrete vector bright-dark solutions and their controllable behaviors in the coupled Ablowitz-Ladik equation with variable coefficients are considered in [27]. The Darboux transformation method with $4 \times 4$ spectral problem is applied to a specific equation and then the explicit solutions of the lattice integrable coupling equation are obtained in [28].

In this paper, we construct N-times DT for NNLS equation with DT method, and obtain n-soliton solutions. In addition, the n-soliton solutions can be reduced to 1, 2, or 3-soliton solutions and some parameters are zero. We derive some novel exact solutions including the bright soliton, breather wave soliton. In particularly, the dynamic features of one-soliton, two-soliton, three-soliton solutions and the elastic interactions between the two solitons are displayed.

The rest of this paper is organized as follows. In Section 2, we construct the 
Darboux transformation for nonlocal nonlinear Schrödinger equation. In Section 3, we give the first- second- and third-order explicit solutions for the nonlocal nonlinear Schrödinger equation. Finally, we give some conclusions.

\section{Darboux Transformation for Nonlocal Nonlinear Schrödinger Equation}

Recently, a novel nonlocal nonlinear Schrödinger (NNLS) equation with the self-induced PT-symmetric potential is presented in the form,

$$
i q_{t}(x, t)-\frac{1}{2} q_{x x}(x, t)-\delta q^{2}(x, t) q^{*}(-x, t)=0,
$$

where the subscript denotes the partial derivative in regard to the variables, the star stands for the complex conjugation. The Lax pairs for Equation (1) can be represented in the form

$$
\varphi_{x}=U \varphi=\left(\begin{array}{cc}
\lambda & q(x, t) \\
-\delta q^{*}(-x, t) & -\lambda
\end{array}\right) \varphi,
$$

and

$$
\varphi_{t}=V \varphi=\left(\begin{array}{cc}
-i \lambda^{2}-\frac{i}{2} \delta q(x, t) q^{*}(-x, t) & -i \lambda q(x, t)-\frac{i}{2} q_{x}(x, t) \\
i \delta \lambda q^{*}(-x, t)+\frac{i}{2} \delta q_{x}^{*}(-x, t) & i \lambda^{2}+\frac{i}{2} \delta q(x, t) q^{*}(-x, t)
\end{array}\right) \varphi
$$

here $q(x, t), q^{*}(-x, t), q_{x}(x, t), q_{x}^{*}(-x, t)$ are potential functions of $x$ and $t$, $\lambda$ is a spectral parameter, $\varphi=\left(\varphi_{1}, \varphi_{2}\right)^{\mathrm{T}}$ is a column vector solution of Eqs.(2) and (3) associated with an eigenvalue $\lambda$ and $i^{2}=-1$.

The aim of this section is to construct DT for NNLS Equations (2) and (3), which are satisfied with the $2 \times 2$ matrix transformation of $\varphi, \tilde{U}$ and $\tilde{V}$. In the following, we construct the $\mathrm{N}$-fold DT and introduce a gauge transformation Tof NNLS equation:

$$
\tilde{\varphi}_{n}=T \varphi_{n}, T=\left(\begin{array}{ll}
T_{11} & T_{12} \\
T_{21} & T_{22}
\end{array}\right) .
$$

The lower form is obtained by compatibility

$$
\begin{gathered}
\varphi_{x}=\tilde{U} \varphi, \tilde{U}=\left(T_{x}+T U\right) T^{-1}, \\
\varphi_{t}=\tilde{V} \varphi, \tilde{V}=\left(T_{t}+T V\right) T^{-1} .
\end{gathered}
$$

If the $\tilde{U}, \tilde{V}$ and $U, V$ have the same types, the system (4) is called Darboux transformation of the NNLS equation. Let $\Psi=\left(\Psi_{1}, \Psi_{2}\right)^{\mathrm{T}}, \phi=\left(\phi_{1}, \phi_{2}\right)^{\mathrm{T}}$ are two basic solutions of the systems (2) and (3), then we give the following linear algebraic systems:

$$
\left\{\begin{array}{l}
\sum_{i=0}^{N-1}\left(A_{11}^{(i)}+A_{12}^{(i)} M_{j}^{(1)}\right) \lambda_{j}^{i}=-\lambda_{j}^{N}, \\
\sum_{i=0}^{N-1}\left(A_{21}^{(i)}+A_{22}^{(i)} M_{j}^{(1)}\right) \lambda_{j}^{i}=-M_{j}^{(1)} \lambda_{j}^{N} .
\end{array}\right.
$$

With 


$$
M_{j}^{(1)}=\frac{\Psi_{2}+v_{j}^{(1)} \phi_{2}}{\Psi_{1}+v_{j}^{(1)} \phi_{1}}, 0 \leq j \leq 2 N,
$$

where $\lambda_{j}$ and $v_{j}^{(k)} \quad\left(i \neq k, \lambda_{i} \neq \lambda_{j}, v_{i}^{(k)} \neq v_{j}^{(k)}, k \neq 1,2\right)$ should choose appropriate parameters, thus the determinants of coefficients for Equation (7) are nonzero. Hereby, we take a $2 \times 2$ matrix $T$ as

$$
\left\{\begin{array}{l}
T_{11}=\lambda^{N}+\sum_{i=0}^{N-1} A_{11}^{(i)} \lambda^{i}, T_{12}=\sum_{i=0}^{N-1} A_{12}^{(i)} \lambda^{i} \\
T_{21}=\sum_{i=0}^{N-1} A_{21}^{(i)} \lambda^{i}, T_{22}=\lambda^{N}+\sum_{i=0}^{N-1} A_{22}^{(i)} \lambda^{i}
\end{array}\right.
$$

where $N$ is a natural number, the $A_{m n}^{i}(m, n=1,2, m \geq 0)$ is the function of $x$ and $t$. Through calculations, we can obtain $\Delta T$ as following

$$
\Delta T=\prod_{j=1}^{2 N}\left(\lambda-\lambda_{j}\right)
$$

which proves that $\lambda_{j}\left(\lambda_{j} \neq 0\right)(j=1,2,3, \cdots, 2 N)$ are $2 N$ roots of $\Delta T$. Based on these conditions, we will proof that the $\tilde{U}$ and $\tilde{V}$ have the same structures as $U$ and $V$, respectively.

Proposition 1. The matrix $\tilde{U}$ defined by (5) has the same type as $U$, that is,

$$
\tilde{U}=\left(\begin{array}{cc}
\lambda & \tilde{q}(x, t) \\
-\delta \tilde{q}^{*}(-x, t) & -\lambda
\end{array}\right),
$$

in which the transformation formulae between old and new potentials are defined by

$$
\left\{\begin{array}{l}
\tilde{q}(x, t)=q(x, t)-2 A_{12}, \\
-\delta \tilde{q}^{*}(-x, t)=-\delta q^{*}(-x, t)+2 A_{21},
\end{array}\right.
$$

the transformations (12) are used to get a Darboux transformation of the spectral problem (5).

Proof. Let $T^{-1}=\frac{T^{*}}{\Delta T}$ with

$$
\left(T_{x}+T U\right) T^{*}=\left(\begin{array}{ll}
B_{11}(\lambda) & B_{12}(\lambda) \\
B_{21}(\lambda) & B_{22}(\lambda)
\end{array}\right),
$$

it is easy to verify that $B_{s l}(1 \leq s, l \leq 2)$ is $2 N$-order or $2 N+1$-order polynomial in $\lambda$.

Through some accurate calculations, $\lambda_{j}(1 \leq j \leq 2)$ is the root of $B_{s l}(1 \leq s, l \leq 2)$. Thus, Equation (13) has the following structure

$$
\left(T_{x}+T U\right) T^{*}=(\Delta T) C(\lambda),
$$

where

$$
C(\lambda)=\left(\begin{array}{cc}
C_{11}^{(1)} \lambda+C_{11}^{(0)} & C_{12}^{(0)} \\
C_{21}^{(0)} & C_{22}^{(1)} \lambda+C_{22}^{(0)}
\end{array}\right),
$$

and $C_{m n}^{(k)}(m, n=1,2, k=0,1)$ satisfy the functions without $\lambda$. Equation (14) can be rewritten as

$$
\left(T_{x}+T U\right)=C(\lambda) T
$$


Through comparing the coefficients of $\lambda$ in Equation (16), we can obtain

$$
\left\{\begin{array}{l}
C_{11}^{(1)}=1, C_{11}^{(0)}=0, C_{12}^{(0)}=q(x, t)-2 A_{12}=\tilde{q}(x, t), \\
C_{21}^{(0)}=-\delta q^{*}(-x, t)+2 A_{21}=-\delta \tilde{q}^{*}(-x, t), C_{22}^{(1)}=-1, C_{22}^{(0)}=0 .
\end{array}\right.
$$

In the following section, we assume that the new matrix $\tilde{U}$ has the same type with $U$, which means that they have the same structures only $q(x, t)$, $q^{*}(-x, t), q_{x}(x, t), q_{x}^{*}(-x, t)$ of $U$ transformed into $\tilde{q}(x, t), \tilde{q}^{*}(-x, t)$, $\tilde{q}_{x}(x, t), \tilde{q}_{x}^{*}(-x, t)$ of $\tilde{U}$. After careful calculation, we compare the ranks of $\lambda^{N}$, and get the objective equations as following:

$$
\left\{\begin{array}{l}
\tilde{q}(x, t)=q(x, t)-2 A_{12}, \\
-\delta \tilde{q}^{*}(-x, t)=-\delta q^{*}(-x, t)+2 A_{21}
\end{array}\right.
$$

from Equations (11) and (12), we know that $\tilde{U}=C(\lambda)$. The proof is completed.

Proposition 2. The matrix $\tilde{V}$ defined by the second expression of (6) has the same form as $V$, in which the old potentials $q$ and $q^{*}$ are mapped into $\tilde{q}$ and $\tilde{q}^{*}$, that is,

$$
\tilde{V}=\left(\begin{array}{cc}
-i \lambda^{2}-\frac{i}{2} \delta \tilde{q}(x, t) \tilde{q}^{*}(-x, t) & -i \lambda \tilde{q}(x, t)-\frac{i}{2} \tilde{q}_{x}(x, t) \\
i \delta \lambda \tilde{q}^{*}(-x, t)+\frac{i}{2} \delta \tilde{q}_{x}^{*}(-x, t) & i \lambda^{2}+\frac{i}{2} \delta \tilde{q}(x, t) \tilde{q}^{*}(-x, t)
\end{array}\right) .
$$

Proof. We suppose the new matrix $\tilde{V}$ also has the same form with $V$. If we obtain the similar relations between $q(x, t), q^{*}(-x, t)$ and $\tilde{q}(x, t), \tilde{q}^{*}(-x, t)$ in Equation (12), we can prove that the gauge transformations under $T$ turn the Lax pairs $U, V$ into new Lax pairs $\tilde{U}, \tilde{V}$ with the same types.

Let $T^{-1}=\frac{T^{*}}{\Delta T}$ and

$$
\left(T_{t}+T V\right) T^{*}=\left(\begin{array}{ll}
E_{11}(\lambda) & E_{12}(\lambda) \\
E_{21}(\lambda) & E_{22}(\lambda)
\end{array}\right)
$$

It is easy to verify that $E_{s l}(1 \leq s, l \leq 2)$ are $N+1$-order or $N+2$-order polynomials in $\lambda$.

Through some calculations, $\lambda_{j}(j=1 \leq j \leq 2)$ are the roots of $E_{s l}(s, l=1 \leq j \leq 2)$. Thus, Equation (20) has the following structure

$$
\left(T_{t}+T V\right) T^{*}=(\Delta T) F(\lambda),
$$

where

$$
F(\lambda)=\left(\begin{array}{cc}
F_{11}^{(2)} \lambda^{2}+F_{11}^{(1)} \lambda+F_{11}^{(0)} & F_{12}^{(1)} \lambda+F_{12}^{(0)} \\
F_{21}^{(1)} \lambda+F_{21}^{(0)} & F_{22}^{(2)} \lambda^{2}+F_{22}^{(1)} \lambda+F_{22}^{(0)}
\end{array}\right),
$$

and $F_{m n}^{(k)}(m, n=1,2, k=0,1)$ satisfy the functions without $\lambda$. According to Equation (21), the following equation is obtained 


$$
\left(T_{t}+T V\right)=F(\lambda) T .
$$

Through comparing the coefficients of $\lambda$ in Equation (23), we get the objective equations as following:

$$
\left\{\begin{aligned}
F_{11}^{(2)} & =-i, F_{11}^{(1)}=0, \\
F_{11}^{(0)} & =-\frac{i}{2} \delta\left(q(x, t) q^{*}(-x, t)-2 q^{*}(-x, t) A_{12}-\frac{2}{\delta} \tilde{q}(x, t) A_{21}\right) \\
& =-\frac{i}{2} \delta \tilde{q}(x, t) \tilde{q}^{*}(-x, t) \\
F_{12}^{(1)} & =-i q(x, t)+2 i A_{12}=-i \tilde{q}(x, t), \\
F_{12}^{(0)} & =-\frac{i}{2}\left(q_{x}(x, t)+2 q(x, t) A_{11}-2 \tilde{q}(x, t) A_{22}\right)=-\frac{i}{2} \tilde{q}_{x}(x, t), \\
F_{21}^{(1)} & =i \delta q^{*}(-x, t)-2 i A_{21}=i \delta \tilde{q}^{*}(-x, t), \\
F_{21}^{(0)} & =\frac{i}{2} \delta\left(q_{x}^{*}(-x, t)+2 q^{*}(-x, t) A_{22}-2 \tilde{q}^{*}(-x, t)\right)=\frac{i}{2} \tilde{q}_{x}^{*}(-x, t), \\
F_{22}^{(2)} & =i, F_{22}^{(1)}=0 \\
F_{22}^{(0)} & =\frac{i}{2} \delta\left(q(x, t) q^{*}(-x, t)-2 q(x, t) A_{21}-2 \delta \tilde{q}^{*}(-x, t)\right)=\frac{i}{2} \delta \tilde{q}(x, t) \tilde{q}^{*}(-x, t)
\end{aligned}\right.
$$

In the above section, we assume the new matrix $\tilde{V}$ has the same type with $V$, which means they have the same structures only $q(x, t), q^{*}(-x, t), q_{x}(x, t)$, $q_{x}^{*}(-x, t)$ of $V$ transformed into $\tilde{q}(x, t), \quad \tilde{q}^{*}(-x, t), \tilde{q}_{x}(x, t), \tilde{q}_{x}^{*}(-x, t)$ of $\tilde{V}$. From Equations (12) and (19), we know that $\tilde{V}=F(\lambda)$. The proof is completed. The transformation matrix $T$ of the nonlocal equation is difficult to construct in making DT, while the transformation matrix $T$ of the local equation is relatively simple.

\section{Explicit Solutions for the Nonlocal Nonlinear Schrödinger Equation}

In this section, we will give some novel explicit solutions of Equation (1) by applying the N-fold DT. Firstly, we give a set of seed solutions $q=0$ and substitute the solution into Equations (2) and (3), it is easy to find two basic solutions for these equations:

$$
\psi(\lambda)=\left(\begin{array}{c}
\mathrm{e}^{\lambda x-i \lambda^{2} t} \\
0
\end{array}\right), \phi(\lambda)=\left(\begin{array}{c}
0 \\
\mathrm{e}^{-\lambda x+i \lambda^{2} t}
\end{array}\right) .
$$

By using Equations (25) and (8), we obtain

$$
M_{j}=\frac{v_{j}^{(i)} \mathrm{e}^{-\lambda x+i \lambda^{2} t}}{\mathrm{e}^{\lambda x-i \lambda^{2} t}}=\mathrm{e}^{-2\left(\lambda_{j} x-i \lambda_{j}^{2} t+F_{j}\right)}
$$

with $v_{j}=\mathrm{e}^{\left(-2 i F_{j}\right)}(1 \leq j \leq 2 N)$.

In order to the expression of N-order DT of Equation (1) and obtain the matrix $T$ 


$$
T=\left(\begin{array}{cc}
\lambda^{N}+\sum_{i=0}^{N-1} A_{11}^{(i)} \lambda^{i} & \sum_{i=0}^{N-1} A_{12}^{(i)} \lambda^{i} \\
\sum_{i=0}^{N-1} A_{21}^{(i)} \lambda^{i} & \lambda^{N}+\sum_{i=0}^{N-1} A_{22}^{(i)} \lambda^{i}
\end{array}\right),
$$

and

$$
\left\{\begin{array}{l}
\lambda_{j}^{N}+\sum_{i=0}^{N-1}\left(A_{11}^{(i)}+M_{j} A_{12}^{(i)}\right) \lambda_{j}^{i}=0, \\
\sum_{i=0}^{N-1} A_{21}^{(i)} \lambda_{j}^{i}+M_{j}\left(\lambda_{j}^{N}+\sum_{i=0}^{N-1} A_{22}^{(i)} \lambda_{j}^{i}\right)=0 .
\end{array}\right.
$$

Solving Equation (7) via the Gramer's rule, we have

$$
A_{12}^{(N)}=\frac{\Delta A_{12}^{(N)}}{\Delta}, A_{21}^{(N)}=\frac{\Delta A_{21}^{(N)}}{\Delta},
$$

with

$\Delta=\left|\begin{array}{cccccccc}1 & \mathrm{e}^{-2\left(\lambda_{1} x-i \lambda_{1}^{2} t+F_{1}\right)} & \lambda_{1} & \lambda_{1} \mathrm{e}^{-2\left(\lambda_{1} x-i \lambda_{1}^{2} t+F_{1}\right)} & \lambda_{1}^{2} & \cdots & \lambda_{1}^{N-1} & \lambda_{1}^{N-1} \mathrm{e}^{-2\left(\lambda_{1} x-i \lambda_{1}^{2} t+F_{1}\right)} \\ 1 & \mathrm{e}^{-2\left(\lambda_{2} x-i \lambda_{2}^{2} t+F_{2}\right)} & \lambda_{2} & \lambda_{2} \mathrm{e}^{-2\left(\lambda_{2} x-i \lambda_{2}^{2} t+F_{2}\right)} & \lambda_{2}^{2} & \cdots & \lambda_{2}^{N-1} & \lambda_{2}^{N-1} \mathrm{e}^{-2\left(\lambda_{2} x-i \lambda_{2}^{2} t+F_{2}\right)} \\ \vdots & \vdots & \vdots & \vdots & \vdots & \ddots & \vdots & \vdots \\ 1 & \mathrm{e}^{-2\left(\lambda_{2 N} x-i \lambda_{2 N}^{2} t+F_{2 N}\right)} & \lambda_{2 N} & \lambda_{2 N} \mathrm{e}^{-2\left(\lambda_{2 N} x-i \lambda_{2 N}^{2} t+F_{2 N}\right)} & \lambda_{2 N}^{2} & \cdots & \lambda_{2 N}^{N-1} & \lambda_{2 N}^{N-1} \mathrm{e}^{-2\left(\lambda_{2 N} x-i \lambda_{2 N}^{2} t+F_{2 N}\right)}\end{array}\right|$,

$\Delta A_{12}=\left|\begin{array}{ccccccc}1 & \lambda_{1} & \lambda_{1} \mathrm{e}^{-2\left(\lambda_{1} x-i \lambda_{1}^{2} t+F_{1}\right)} & \lambda_{1}^{2} & \cdots & \lambda_{1}^{N-1} \mathrm{e}^{-2\left(\lambda_{1} x-i \lambda_{1}^{2} t+F_{1}\right)} & -\lambda_{1}^{N} \\ 1 & \lambda_{2} & \lambda_{2} \mathrm{e}^{-2\left(\lambda_{2} x-i \lambda_{2}^{2} t+F_{2}\right)} & \lambda_{2}^{2} & \cdots & \lambda_{2}^{N-1} \mathrm{e}^{-2\left(\lambda_{2} x-i \lambda_{2}^{2} t+F_{2}\right)} & -\lambda_{2}^{N} \\ \vdots & \vdots & \vdots & \vdots & \ddots & \vdots & \vdots \\ 1 & \lambda_{2 N} & \lambda_{2 N} \mathrm{e}^{-2\left(\lambda_{2 N} x-i \lambda_{2 N}^{2} t+F_{2 N}\right)} & \lambda_{2 N}^{N-1} & \cdots & \lambda_{2 N}^{N-1} \mathrm{e}^{-2\left(\lambda_{2 N} x-i \lambda_{2 N}^{2} t+F_{2 N}\right)} & -\lambda_{2 N}^{N}\end{array}\right|$,

$\Delta A_{21}=\left|\begin{array}{cccccc}\mathrm{e}^{-2\left(\lambda_{1} x-i \lambda_{1}^{2} t+F_{1}\right)} & \lambda_{1} & \lambda_{1} \mathrm{e}^{-2\left(\lambda_{1} x-i \lambda_{1}^{2} t+F_{1}\right)} & \lambda_{1}^{2} & \cdots & -\lambda_{1}^{N-1} \mathrm{e}^{-2\left(\lambda_{1} x-i \lambda_{1}^{2} t+F_{1}\right)} \\ \mathrm{e}^{-2\left(\lambda_{2} x-i \lambda_{2}^{2} t+F_{2}\right)} & \lambda_{2} & \lambda_{2} \mathrm{e}^{-2\left(\lambda_{2} x-i \lambda_{2}^{2} t+F_{2}\right)} & \lambda_{2}^{2} & \cdots & -\lambda_{2}^{N-1} \mathrm{e}^{-2\left(\lambda_{2} x-i \lambda_{2}^{2} t+F_{2}\right)} \\ \vdots & \vdots & \vdots & \vdots & \ddots & \vdots \\ \mathrm{e}^{-2\left(\lambda_{2 N} x-i \lambda_{2 N}^{2} t+F_{2 N}\right)} & \lambda_{2 N} & \lambda_{2 N} \mathrm{e}^{-2\left(\lambda_{2 N} x-i \lambda_{2 N}^{2} t+F_{2 N}\right)} & \lambda_{2 N}^{2} & \cdots & -\lambda_{2 N}^{N-1} \mathrm{e}^{-2\left(\lambda_{2 N} x-i \lambda_{2 N}^{2} t+F_{2 N}\right)}\end{array}\right| \cdot(30)$

Based on Equations (8) and (26), we can obtain the following systems

$$
A_{12}^{(N)}=\frac{\Delta A_{12}^{(N)}}{\Delta}, A_{21}^{(N)}=\frac{A_{21}^{(N)}}{\Delta} .
$$

Using Equations (4), (18) and (29), we can derive the new the formula of $\mathrm{N}$-soliton solutions for NNLS equation

$$
\left\{\begin{array}{l}
\tilde{q}(x, t)=-2 \frac{\Delta A_{12}^{(N)}}{\Delta}, \\
-\delta \tilde{q}^{*}(-x, t)=2 \frac{\Delta A_{21}^{(N)}}{\Delta} .
\end{array}\right.
$$

To understand solutions (32), we consider $N=1,2,3$ separately and plot their structure figures as Figures 1-3.

1) We take $N=1$ with $\lambda=\lambda_{j}(j=1,2)$. Solving (7), we can yield,

$$
A_{12}^{(1)}=\frac{\Delta A_{12}^{(1)}}{\Delta}, A_{21}^{(1)}=\frac{\Delta A_{21}^{(1)}}{\Delta},
$$




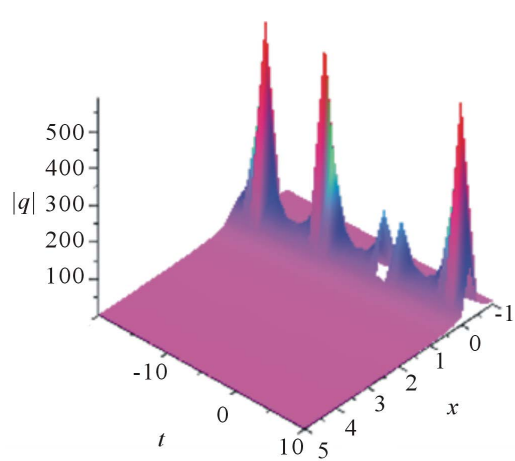

(a)

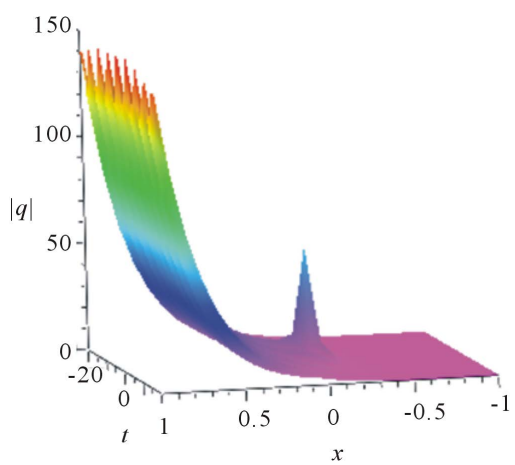

(c)

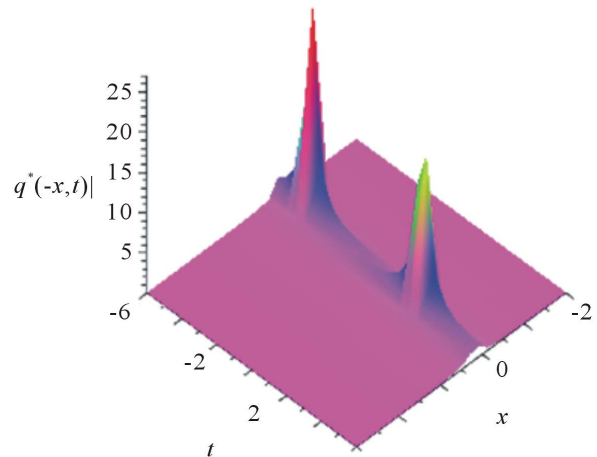

(b)

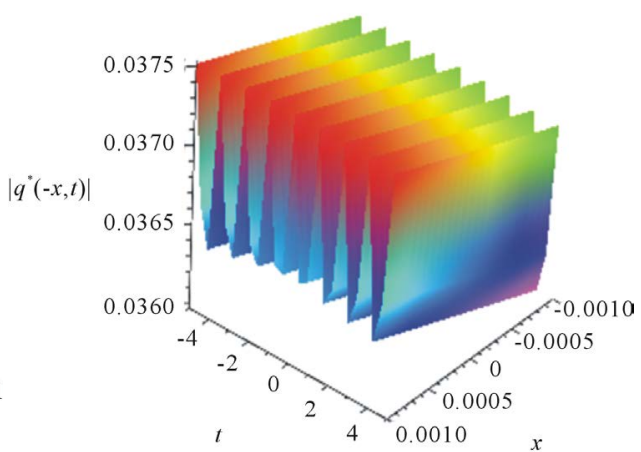

(d)

Figure 1. Profiles of (a), (c) and (b),(d) the intensity distributions $\tilde{q}$ and $\tilde{q}^{*}$ with $\tilde{q}=|q|, \quad \tilde{q}^{*}=\left|q^{*}\right|$, and $\delta=1$. The parameters of (a) and (b) are $\lambda_{1}=0.01 i, \lambda_{2}=0.02 i$, $F_{1}=1, F_{2}=1$. The parameters of $(\mathrm{c})$ and $(\mathrm{d})$ are $\lambda_{1}=2, \lambda_{2}=3, F_{1}=0, F_{2}=2$.

with

$$
\begin{aligned}
& \Delta=\left|\begin{array}{cc}
1 & \mathrm{e}^{-2\left(\lambda_{1} x-i \lambda_{1}^{2} t+F_{1}\right)} \\
1 & \mathrm{e}^{-2\left(\lambda_{2} x-i \lambda_{2}^{2} t+F_{2}\right)}
\end{array}\right|, \Delta A_{12}=\left|\begin{array}{cc}
1 & -\lambda_{1} \\
1 & -\lambda_{2}
\end{array}\right|, \\
& \Delta A_{21}=\left|\begin{array}{cc}
-\lambda_{1} \mathrm{e}^{-2\left(\lambda_{1} x-i \lambda_{1}^{2} t+F_{1}\right)} & \mathrm{e}^{-2\left(\lambda_{1} x-i \lambda_{1}^{2} t+F_{1}\right)} \\
-\lambda_{2} \mathrm{e}^{-2\left(\lambda_{2} x-i \lambda_{2}^{2} t+F_{2}\right)} & \mathrm{e}^{-2\left(\lambda_{2} x-i \lambda_{2}^{2} t+F_{2}\right)}
\end{array}\right| .
\end{aligned}
$$

In this way, the nonlocal one-soliton solutions of NNLS equation (1) are obtained as following:

$$
\left\{\begin{array}{l}
\tilde{q}(x, t)=-2 \frac{\Delta A_{12}^{(1)}}{\Delta}, \\
-\delta \tilde{q}^{*}(-x, t)=2 \frac{\Delta A_{21}^{(1)}}{\Delta} .
\end{array}\right.
$$

2) We take $N=2$ in the $N$-times DT with $\lambda=\lambda_{j}(j=1,2,3,4)$. The linear algebraic system (7) lead to the following solutions,

$$
A_{12}^{(2)}=\frac{\Delta A_{12}^{(2)}}{\Delta}, A_{21}^{(2)}=\frac{\Delta A_{21}^{(2)}}{\Delta},
$$

with 


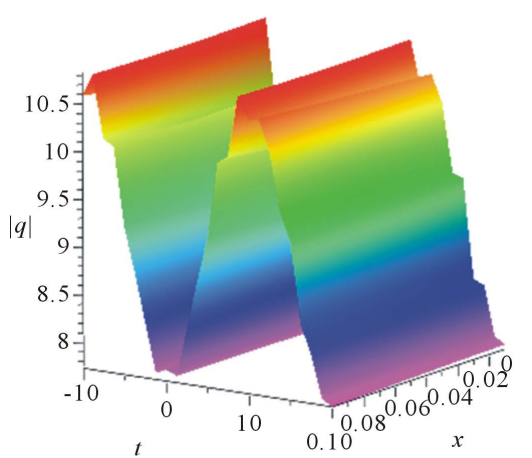

(a)

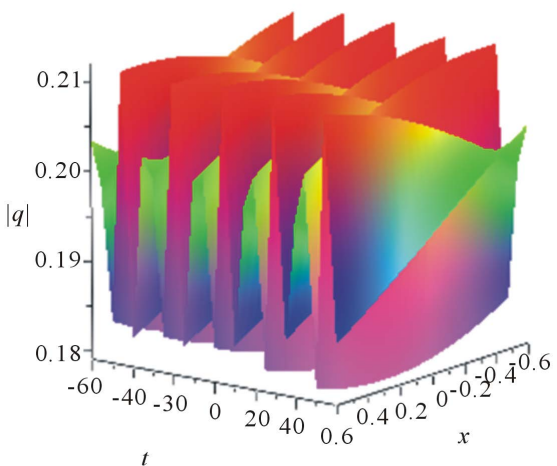

(c)

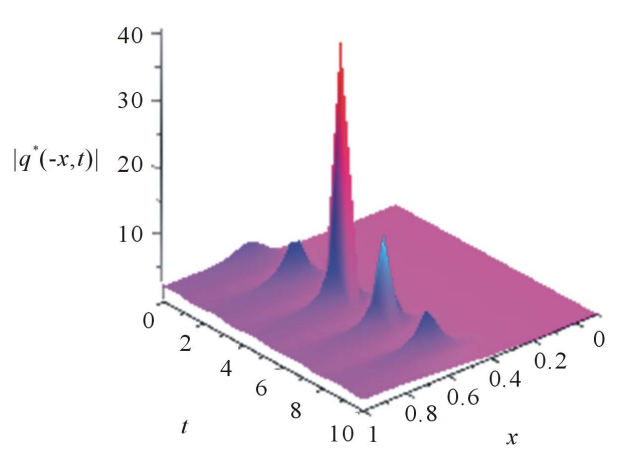

(b)

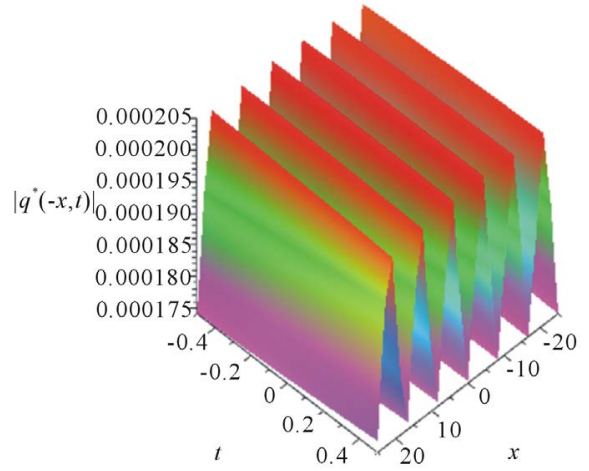

(d)

Figure 2. (Color online) Profiles of (a), (c) and (b), (d) the intensity distributions $\tilde{\tilde{q}}$ and $\tilde{\tilde{q}}^{*}$ with $\tilde{\tilde{q}}=|q|, \quad \tilde{\tilde{q}}^{*}=\left|q^{*}\right|$, and $\delta=1$. The parameters of (a) and (b) are $\lambda_{1}=0.02$, $\lambda_{2}=3, \lambda_{3}=0.4, \lambda_{4}=0.5, F_{1}=0.1, F_{2}=2, F_{3}=0, F_{4}=0$. The parameters of $(\mathrm{c})$ and (d) are $\lambda_{1}=0.02 i, \lambda_{2}=0.03 i, \lambda_{3}=0.4 i, \lambda_{4}=0.5 i, F_{1}=0, F_{2}=2, F_{3}=3$, $F_{4}=0$.

$$
\begin{aligned}
& \Delta=\left|\begin{array}{llll}
1 & \mathrm{e}^{-2\left(\lambda_{1} x-i \lambda_{1}^{2} t+F_{1}\right)} & \lambda_{1} & \lambda_{1} \mathrm{e}^{-2\left(\lambda_{1} x-i \lambda_{1}^{2} t+F_{1}\right)} \\
1 & \mathrm{e}^{-2\left(\lambda_{2} x-i \lambda_{2}^{2} t+F_{2}\right)} & \lambda_{2} & \lambda_{2} \mathrm{e}^{-2\left(\lambda_{2} x-i \lambda_{2}^{2} t+F_{2}\right)} \\
1 & \mathrm{e}^{-2\left(\lambda_{3} x-i \lambda_{3}^{2} t+F_{3}\right)} & \lambda_{3} & \lambda_{3} \mathrm{e}^{-2\left(\lambda_{3} x-i \lambda_{3}^{2} t+F_{3}\right)} \\
1 & \mathrm{e}^{-2\left(\lambda_{4} x-i \lambda_{4}^{2} t+F_{4}\right)} & \lambda_{4} & \lambda_{4} \mathrm{e}^{-2\left(\lambda_{4} x-i \lambda_{4}^{2} t+F_{4}\right)}
\end{array}\right|, \\
& \Delta A_{12}=\left|\begin{array}{llll}
1 & \lambda_{1} & \mathrm{e}^{-2\left(\lambda_{1} x-i \lambda_{1}^{2} t+F_{1}\right)} & -\lambda_{1}^{2} \\
1 & \lambda_{2} & \mathrm{e}^{-2\left(\lambda_{2} x-i \lambda_{2}^{2} t+F_{2}\right)} & -\lambda_{2}^{2} \\
1 & \lambda_{3} & \mathrm{e}^{-2\left(\lambda_{3} x-i \lambda_{3}^{2} t+F_{3}\right)} & -\lambda_{3}^{2} \\
1 & \lambda_{4} & \mathrm{e}^{-2\left(\lambda_{4} x-i \lambda_{4}^{2} t+F_{4}\right)} & -\lambda_{4}^{2}
\end{array}\right|, \\
& \Delta A_{21}=\left|\begin{array}{llll}
\mathrm{e}^{-2\left(\lambda_{1} x-i \lambda_{1}^{2} t+F_{1}\right)} & \lambda_{1} & \lambda_{1} \mathrm{e}^{-2\left(\lambda_{1} x-i \lambda_{1}^{2} t+F_{1}\right)} & -\lambda_{1}^{2} \mathrm{e}^{-2\left(\lambda_{1} x-i \lambda_{1}^{2} t+F_{1}\right)} \\
\mathrm{e}^{-2\left(\lambda_{2} x-i \lambda_{2}^{2} t+F_{2}\right)} & \lambda_{2} & \lambda_{2} \mathrm{e}^{-2\left(\lambda_{2} x-i \lambda_{2}^{2} t+F_{2}\right)} & -\lambda_{2}^{2} \mathrm{e}^{-2\left(\lambda_{2} x-i \lambda_{2}^{2} t+F_{2}\right)} \\
\mathrm{e}^{-2\left(\lambda_{3} x-i \lambda_{3}^{2} t+F_{3}\right)} & \lambda_{3} & \lambda_{3} \mathrm{e}^{-2\left(\lambda_{3} x-i \lambda_{3}^{2} t+F_{3}\right)} & -\lambda_{3}^{2} \mathrm{e}^{-2\left(\lambda_{3} x-i \lambda_{3}^{2} t+F_{3}\right)} \\
\mathrm{e}^{-2\left(\lambda_{4} x-i \lambda_{4}^{2} t+F_{4}\right)} & \lambda_{4} & \lambda_{4} \mathrm{e}^{-2\left(\lambda_{4} x-i \lambda_{4}^{2} t+F_{4}\right)} & -\lambda_{4}^{2} \mathrm{e}^{-2\left(\lambda_{4} x-i \lambda_{4}^{2} t+F_{4}\right)}
\end{array}\right| .
\end{aligned}
$$


In this way, the nonlocal two-soliton solutions of NNLS Equation (1) are obtained as following:

$$
\left\{\begin{array}{l}
\tilde{q}(x, t)=-2 \frac{\Delta A_{12}^{(2)}}{\Delta}, \\
-\delta \tilde{q}^{*}(-x, t)=2 \frac{\Delta A_{21}^{(2)}}{\Delta} .
\end{array}\right.
$$

3) We take $N=3$ in the $N$-times DT with $\lambda=\lambda_{j}(j=1,2,3,4,5,6)$. The linear algebraic System (7) lead to solutions as following,

$$
A_{12}^{(3)}=\frac{\Delta A_{12}^{(3)}}{\Delta}, A_{21}^{(3)}=\frac{\Delta A_{21}^{(3)}}{\Delta},
$$

with

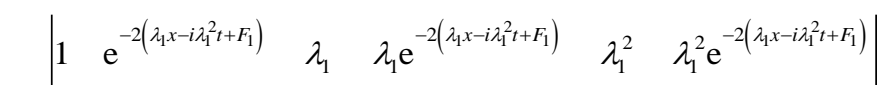

$$
\begin{aligned}
& 1 \quad \mathrm{e}^{-2\left(\lambda_{2} x-i \lambda_{2}^{2} t+F_{2}\right)} \quad \lambda_{2} \quad \lambda_{2} \mathrm{e}^{-2\left(\lambda_{2} x-i \lambda_{2}^{2} t+F_{2}\right)} \quad \lambda_{2}^{2} \quad \lambda_{2}^{2} \mathrm{e}^{-2\left(\lambda_{2} x-i \lambda_{2}^{2} t+F_{2}\right)}
\end{aligned}
$$

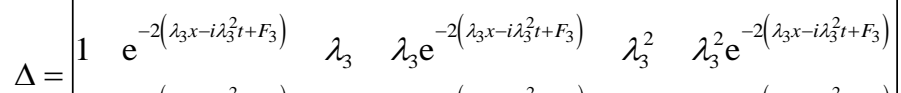

$$
\begin{aligned}
& 1 \quad \mathrm{e}^{-2\left(\lambda_{4} x-i \lambda_{4}^{2} t+F_{4}\right)} \quad \lambda_{4} \quad \lambda_{4} \mathrm{e}^{-2\left(\lambda_{4} x-i \lambda_{4}^{2} t+F_{4}\right)} \quad \lambda_{4}^{2} \quad \lambda_{4}^{2} \mathrm{e}^{-2\left(\lambda_{4} x-i \lambda_{4}^{2} t+F_{4}\right)} \\
& 1 \quad \mathrm{e}^{-2\left(\lambda_{5} x-i \lambda_{5}^{2} t+F_{5}\right)} \quad \lambda_{5} \quad \lambda_{5} \mathrm{e}^{-2\left(\lambda_{5} x-i \lambda_{5}^{2} t+F_{5}\right)} \quad \lambda_{5}^{2} \quad \lambda_{5}^{2} \mathrm{e}^{-2\left(\lambda_{5} x-i \lambda_{5}^{2} t+F_{5}\right)} \\
& 1 \quad \mathrm{e}^{-2\left(\lambda_{6} x-i \lambda_{6}^{2} t+F_{6}\right)} \quad \lambda_{6} \quad \lambda_{6} \mathrm{e}^{-2\left(\lambda_{6} x-i \lambda_{6}^{2} t+F_{6}\right)} \quad \lambda_{6}^{2} \quad \lambda_{6}^{2} \mathrm{e}^{-2\left(\lambda_{6} x-i \lambda_{6}^{2} t+F_{6}\right)} \mid \\
& \Delta A_{12}=\left|\begin{array}{cccccc}
1 & \lambda_{1} & \mathrm{e}^{-2\left(\lambda_{1} x-i \lambda_{1}^{2} t+F_{1}\right)} & \lambda_{1}^{2} & \lambda_{1}^{2} \mathrm{e}^{-2\left(\lambda_{1} x-i \lambda_{1}^{2} t+F_{1}\right)} & -\lambda_{1}^{3} \\
1 & \lambda_{2} & \mathrm{e}^{-2\left(\lambda_{2} x-i \lambda_{2}^{2} t+F_{2}\right)} & \lambda_{2}^{2} & \lambda_{2}^{2} \mathrm{e}^{-2\left(\lambda_{2} x-i \lambda_{2}^{2} t+F_{2}\right)} & -\lambda_{2}^{3} \\
1 & \lambda_{3} & \mathrm{e}^{-2\left(\lambda_{3} x-i \lambda_{3}^{2} t+F_{3}\right)} & \lambda_{3}^{2} & \lambda_{3}^{2} \mathrm{e}^{-2\left(\lambda_{3} x-i \lambda_{3}^{2} t+F_{3}\right)} & -\lambda_{3}^{3} \\
1 & \lambda_{4} & \mathrm{e}^{-2\left(\lambda_{4} x-i \lambda_{4}^{2} t+F_{4}\right)} & \lambda_{4}^{2} & \lambda_{4}^{2} \mathrm{e}^{-2\left(\lambda_{4} x-i \lambda_{4}^{2} t+F_{4}\right)} & -\lambda_{4}^{3} \\
1 & \lambda_{5} & \mathrm{e}^{-2\left(\lambda_{5} x-i \lambda_{5}^{2} t+F_{5}\right)} & \lambda_{5}^{2} & \lambda_{5}^{2} \mathrm{e}^{-2\left(\lambda_{5} x-i \lambda_{5}^{2} t+F_{5}\right)} & -\lambda_{5}^{3} \\
1 & \lambda_{6} & \mathrm{e}^{-2\left(\lambda_{6} x-i \lambda_{6}^{2} t+F_{6}\right)} & \lambda_{6}^{2} & \lambda_{6}^{2} \mathrm{e}^{-2\left(\lambda_{6} x-i \lambda_{6}^{2} t+F_{6}\right)} & -\lambda_{6}^{3}
\end{array}\right|, \\
& \Delta A_{21}=\left|\begin{array}{cccccc}
\mathrm{e}^{-2\left(\lambda_{1} x-i \lambda_{1}^{2} t+F_{1}\right)} & \lambda_{1} & \lambda_{1} \mathrm{e}^{-2\left(\lambda_{1} x-i \lambda_{1}^{2} t+F_{1}\right)} & \lambda_{1}^{2} & \lambda_{1}^{2} \mathrm{e}^{-2\left(\lambda_{1} x-i \lambda_{1}^{2} t+F_{1}\right)} & -\lambda_{1}^{3} \mathrm{e}^{-2\left(\lambda_{1} x-i \lambda_{1}^{2} t+F_{1}\right)} \\
\mathrm{e}^{-2\left(\lambda_{2} x-i \lambda_{2}^{2} t+F_{2}\right)} & \lambda_{2} & \lambda_{2} \mathrm{e}^{-2\left(\lambda_{2} x-i \lambda_{2}^{2} t+F_{2}\right)} & \lambda_{2}^{2} & \lambda_{2}^{2} \mathrm{e}^{-2\left(\lambda_{2} x-i \lambda_{2}^{2} t+F_{2}\right)} & -\lambda_{2}^{3} \mathrm{e}^{-2\left(\lambda_{2} x-i \lambda_{2}^{2} t+F_{2}\right)} \\
\mathrm{e}^{-2\left(\lambda_{3} x-i \lambda_{3}^{2} t+F_{3}\right)} & \lambda_{3} & \lambda_{3} \mathrm{e}^{-2\left(\lambda_{3} x-i \lambda_{3}^{2} t+F_{3}\right)} & \lambda_{3}^{2} & \lambda_{3}^{2} \mathrm{e}^{-2\left(\lambda_{3} x-i \lambda_{3}^{2} t+F_{3}\right)} & -\lambda_{3}^{3} \mathrm{e}^{-2\left(\lambda_{3} x-i \lambda_{3}^{2} t+F_{3}\right)} \\
\mathrm{e}^{-2\left(\lambda_{5} x-i \lambda_{4}^{2} t+F_{4}\right)} & \left.\lambda_{4} t+F_{5}\right) & \lambda_{4} \mathrm{e}^{-2\left(\lambda_{4} x-i \lambda_{4}^{2} t+F_{4}\right)} & \lambda_{4}^{2} & \lambda_{4}^{2} \mathrm{e}^{-2\left(\lambda_{4} x-i \lambda_{4}^{2} t+F_{4}\right)} & -\lambda_{4}^{3} \mathrm{e}^{-2\left(\lambda_{4} x-i \lambda_{4}^{2} t+F_{4}\right)} \\
\mathrm{e}^{-2\left(\lambda_{6} x-i \lambda_{6}^{2} t+F_{6}\right)} & \lambda_{5} \mathrm{e}^{-2\left(\lambda_{5} x-i \lambda_{5}^{2} t+F_{5}\right)} & \lambda_{5}^{2} & \lambda_{5}^{2} \mathrm{e}^{-2\left(\lambda_{5} x-i \lambda_{5}^{2} t+F_{5}\right)} & -\lambda_{5}^{3} \mathrm{e}^{-2\left(\lambda_{5} x-i \lambda_{5}^{2} t+F_{5}\right)} \\
\lambda_{6} & \lambda_{6} \mathrm{e}^{-2\left(\lambda_{6} x-i \lambda_{6}^{2} t+F_{6}\right)} & \lambda_{6}^{2} & \lambda_{6}^{2} \mathrm{e}^{-2\left(\lambda_{6} x-i \lambda_{6}^{2} t+F_{6}\right)} & -\lambda_{6}^{3} \mathrm{e}^{-2\left(\lambda_{6} x-i \lambda_{6}^{2} t+F_{6}\right)}
\end{array}\right| .
\end{aligned}
$$

In this way, the nonlocal three-soliton solutions of NNLS Equation (1) are obtained as below:

$$
\left\{\begin{array}{l}
\tilde{q}(x, t)=-2 \frac{\Delta A_{12}^{(3)}}{\Delta}, \\
-\delta \tilde{q}^{*}(-x, t)=2 \frac{\Delta A_{21}^{(3)}}{\Delta},
\end{array}\right.
$$




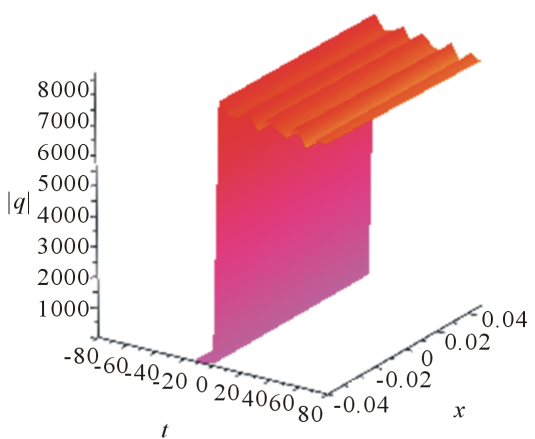

(a)

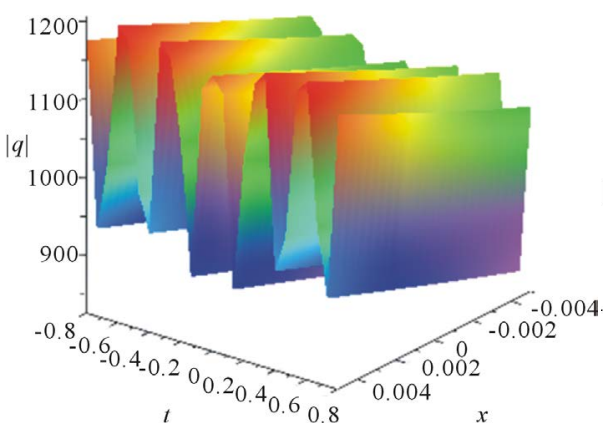

(c)

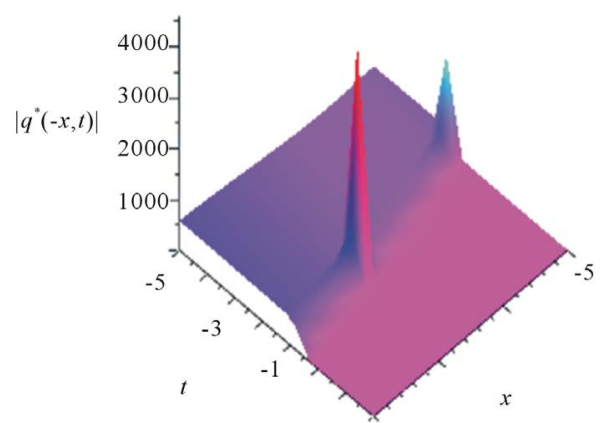

(b)

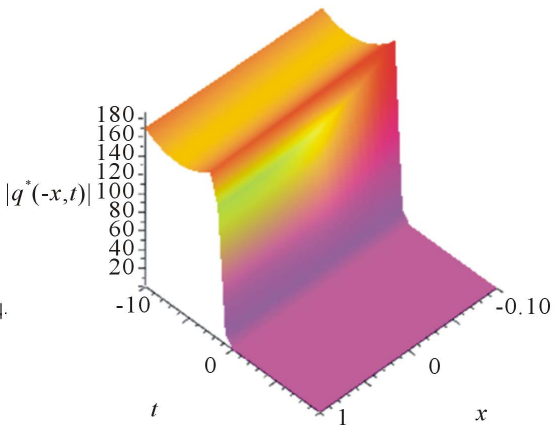

(d)

Figure 3. Profiles of (a), (c) and (b), (d) the intensity distribution $\tilde{\tilde{\tilde{q}}}$ and $\tilde{\tilde{q}}^{*}$ with $|\tilde{\tilde{\tilde{q}}}|=|q|, \quad\left|\tilde{\tilde{\tilde{q}}}^{*}\right|=\left|q^{*}\right|$, let's get $\delta=1$. The parameters of (a) and (b) are $\lambda_{1}=0.1 i$, $\lambda_{2}=0.2 i, \quad \lambda_{3}=3 i, \quad \lambda_{4}=0.4+4 i, \quad \lambda_{5}=0.5+5 i, \quad \lambda_{6}=0.6+6 i, \quad F_{1}=0, \quad F_{2}=0.2$, $F_{3}=3, F_{4}=0.4, F_{5}=5, F_{6}=6$. The parameters of (c) are $\lambda_{1}=0.01, \lambda_{2}=0.02$, $\lambda_{3}=0.03, \lambda_{4}=0.04, \lambda_{5}=5, \lambda_{6}=6, F_{1}=1, F_{2}=2, F_{3}=3, F_{4}=4, F_{5}=0.5$, $F_{6}=0$. The parameters of $(\mathrm{d})$ are $\lambda_{1}=0.1 i, \lambda_{2}=0.2 i, \lambda_{3}=3 i, \lambda_{4}=0.4+4 i$, $\lambda_{5}=0.5+5 i, \quad \lambda_{6}=0.6+6 i, \quad F_{1}=0.1, \quad F_{2}=0.2 i, \quad F_{3}=3, \quad F_{4}=0.4, \quad F_{5}=5, F_{6}=6$.

which are novel soliton solutions including the bright soliton, breather wave soliton. The three-soliton solutions of NNLS Equation (1) are different from the previous works.

Figures 1-3 exhibit the exact one-, two- and three-soliton solutions of NNLS Equation (1). Figure 2 shows the interactions among two solitons with different amplitudes for solution (38). Figure 3 shows the collision interactions among three-solitons with different amplitudes for solution (41). The structure of the nonlocal solution is richer than structure of the local solution, for example the (b) in Figure 1 does not appear in the local area, etc.

\section{Conclusion}

In this paper, we have constructed DT for a new NNLS equation with the self-induced PT-symmetric potential. Selecting the appropriate parameters, we give the expressions of $\mathrm{N}$-soliton solutions, and give 1-, 2- and 3-order soliton solutions. By solving the NNLS equation, we find that it is quite different from the solution of the nonlinear local NLS equation. Moreover, we also study the 
dynamical behaviors of these solutions. These results can explain some physical phenomena. The method is also appropriate for some other nonlinear nonlocal soliton equations in physics and mathematics. In the future work, we want to consider the initial solution besides zero, and try to obtain the DT of the coupled nonlocal equation.

\section{Acknowledgements}

This work was supported by the Natural Science Foundation of Liaoning Province, China (Grant No. 201602678).

\section{References}

[1] Ablowitz, M.J. and Musslimani, Z.H. (2013) Integrable Nonlocal Nonlinear Schrödinger Equation. Physical Review Letters, 110, Article ID: 064105. https://doi.org/10.1103/PhysRevLett.110.064105

[2] Xu, T., Li, H., Zhang, H., Li, M. and Lan, S. (2017) Darboux Transformation and Analytic Solutions of the Discrete PT-Symmetric Nonlocal Nonlinear Schrodinger Equation. Applied Mathematics Letters, 63, 88-94. https://doi.org/10.1016/j.aml.2016.07.024

[3] Fokas, A.S. (2016) Integrable Multidimensional Versions of the Nonlocal Nonlinear Schrödinger Equation. Nonlinearity, 29, 319-324.

https://doi.org/10.1088/0951-7715/29/2/319

[4] Li, M. and Xu, T. (2015) Dark and Antidark Soliton Interactions in the Nonlocal Nonlinear Schrödinger Equation with the Self-Induced Parity-Time-Symmetric Potential. Physical Review E, 91, Article ID: 033202. https://doi.org/10.1103/PhysRevE.91.033202

[5] Ye, G. and Wong, K.W. (2012) An Efficient Chaotic Image Encryption Algorithm Based on a Generalized Arnold Map. Nonlinear Dynamics, 69, 2079-2087.

https://doi.org/10.1007/s11071-012-0409-Z

[6] Guo, R., Tian, B. and Wang, L. (2012) Soliton Solutions for the Reduced Maxwell-Bloch System in Nonlinear Optics via the n-Fold Darboux Transformation. Nonlinear Dynamics, 69, 2009-2020. https://doi.org/10.1007/s11071-012-0403-5

[7] Ablowitz, M.J. and Clarkson, P.A. (1991) Solitons, Nonlinear Evolution Equations and Inverse Scattering. Cambridge University Press, Cambridge. https://doi.org/10.1017/CBO9780511623998

[8] Gerdjikov, V.S. (2017) Bose-Einstein Condensates and Spectral Properties of Multicomponent Nonlinear Schrödinger Equations. Discrete and Continuous Dynamical Systems-Series $S$ (DCDS-S), 4, 1181-1197. https://doi.org/10.3934/dcdss.2011.4.1181

[9] Gu, C.H., Guo, B.L. and Li, Y.S. (1990) Solition Theory and Its Application. Zhejiang Press, Hangzhou.

[10] Cao, C.W. (1989) Nonlinearization of the Lax System for AKNS Hierarchy. Science in China Series A: Mathematics Physics Astronomy, 7, 701-707.

[11] Cao, C. (2002) Algebro-Geometric Solution of the $2+1$ Dimensional Burgers Equation with a Discrete Variable. Journal of Mathematical Physics, 43, 621-643. https://doi.org/10.1063/1.1415427

[12] Hirota, R. (2004) Direct Methods in Solition Theory. Cambridge University Press, Cambridge. https://doi.org/10.1017/CBO9780511543043 
[13] Hu, X.B. and Ma, W. X. (2002) Application of Hirota's Bilinear Formalism to the Toeplitz Lattice-Some Special Soliton-Like Solutions. Physics Letters A, 293, 161-165. https://doi.org/10.1016/S0375-9601(01)00850-7

[14] Wang, M.L. (1995) Solitary Wave Solutions for Variant Boussinesq Equations. Physics Letters A, 199, 169-172. https://doi.org/10.1016/0375-9601(95)00092-H

[15] Fan, E.G. and Zhang, H.Q. (1999) Some New Applications of Homogeneous Balance Method. Acta Mathematieaentia, 19, 286-292.

[16] Hu, X.B. (1994) Nonlinear Superposition Formulae for the Differential-Difference Analogue of the $\mathrm{kdv}$ Equation and Two-Dimensional Toda Equation. Journal of Physics A General Physics, 27, 201. https://doi.org/10.1088/0305-4470/27/1/014

[17] Gu, C.H. and Hu, H.S. (1986) A Unified Explicit form of Bäcklund Transformations for Generalized Hierarchies of kdv Equations. Letters in Mathematical Physics, 11, 325-335. https://doi.org/10.1007/BF00574158

[18] Li, Y.S. and Zhang, J.E. (2001) Darboux Transformations of Classical Boussinesq System and Its Multi-Soliton Solutions. Physics Letters A, 284, 253-258. https://doi.org/10.1016/S0375-9601(01)00331-0

[19] Zhao, Y. (2012) N-Fold Darboux Transformation for a Nonlinear Evolution Equation. Applied Mathematics, 3, 943-948. https://doi.org/10.4236/am.2012.38141

[20] Neugebauer, G. and Kramer, D. (1983) Einstein-Maxwell Solitons. Journal of Physics A General Physics, 16, 1927. https://doi.org/10.1088/0305-4470/16/9/017

[21] Surhone, L.M., Timpledon, M.T. and Marseken, S.F. (2010) University of Science and Technology of China.

[22] Fan, E.G. (2000) Darboux Transformation and Soliton-Like Solutions for the Gerdjikov-Ivanov Equation. Journal of Physics A General Physics, 33, 6925. https://doi.org/10.1088/0305-4470/33/39/308

[23] Yu, F. (2017) Dynamics of Nonautonomous Discrete Rogue Wave Solutions for an Ablowitz-Musslimani Equation with Pt-Symmetric Potential. Chaos, 27, Article ID: 023108. https://doi.org/10.1063/1.4975763

[24] Yu, F., Feng, L. and Li, L. (2017) Darboux Transformations for Super-Schrödinger Equation, Super-Dirac Equation and Their Exact Solutions. Nonlinear Dynamics, 88, 1257-1271. https://doi.org/10.1007/s11071-016-3308-x

[25] Li, L. and Yu, F. (2017) Non-Autonomous Multi-Rogue Waves for Spin-1 Coupled Nonlinear Gross-Pitaevskii Equation and Management by External Potentials. Scientific Reports, 7, Article No. 10638. https://doi.org/10.1038/s41598-017-10205-4

[26] Yu, F. and Li, L. (2017) Vector Dark and Bright Soliton Wave Solutions and Collisions for Spin-1 Bose-Einstein Condensate. Nonlinear Dynamics, 87, 2697-2713. https://doi.org/10.1007/s11071-016-3221-3

[27] Li, L. and Yu, F. (2017) Discrete Bright-Dark Soliton Solutions and Parameters Controlling for the Coupled Ablowitz-Ladik Equation. Nonlinear Dynamics, 89, 2403-2414. https://doi.org/10.1007/s11071-017-3593-Z

[28] Yu, F. and Feng, S. (2017) Explicit Solution and Darboux Transformation for a New Discrete Integrable Soliton Hierarchy with $4 \times 4$ Lax Pairs. Mathematical Methods in the Applied Sciences, 40, 5515-5525. https://doi.org/10.1002/mma.4406 